\title{
Intravenous Vancomycin Dosing in the Elderly: A Focus on Clinical Issues and Practical Application
}

\author{
Katie E. Barber $^{1} \cdot$ Allison M. Bell ${ }^{1} \cdot$ Kayla R. Stover $^{1,2} \cdot$ Jamie L. Wagner $^{1}$
}

Published online: 22 November 2016

(C) The Author(s) 2016. This article is published with open access at Springerlink.com

\begin{abstract}
The elderly population can be divided into three distinct age groups: 65-74 years (young-old), 75-84 years (middle-old), and 85+ years (old-old). Despite evidence of a shift in leading causes for mortality in the elderly from infectious diseases to chronic conditions, infections are still a serious cause of death in this population. These patients are at increased risk due to weakened immune systems, an increased prevalence of underlying comorbidities, and decreased physiologic reserves to fight infection. Additionally, elderly patients, especially adults in institutional settings, are at an increased risk of colonization and subsequent infection with methicillin-resistant Staphylococcus aureus at a rate that is five times higher than in younger individuals, causing an increase in empiric and definitive vancomycin use. Elderly patients have unique characteristics that make dosing vancomycin a challenge for clinicians, such as increased volume of distribution and decreased renal function. Using the best available evidence, it is recommended to initiate lower empiric maintenance doses and monitor vancomycin serum concentrations earlier than steady state to accurately calculate drug elimination and make appropriate dose adjustments.
\end{abstract}

Jamie L. Wagner

jwagner@umc.edu

1 Department of Pharmacy Practice, University of Mississippi School of Pharmacy, 2500 N. State Street, Jackson, MS 39216, USA

2 Department of Medicine-Infectious Diseases, University of Mississippi Medical Center, 2500 N. State Street, Jackson, MS 39216, USA

\section{Key Points}

Elderly patients are at increased risk for infection, especially with methicillin-resistant Staphylococcus aureus.

Debate still exists over how to calculate creatinine clearance in elderly patients.

Dosing vancomycin is challenging in elderly patients, and lower empiric maintenance dosing with earlier concentration monitoring is advised.

\section{Introduction}

While there has been a shift in leading causes of mortality in the elderly from infectious diseases to chronic conditions, infections are still a serious cause of death in this population [1]. The most common cause of hospitalization in nursing home patients is infection, and almost $14 \%$ of elderly patients admitted to the hospital are admitted for infection [2]. Infectious diseases-related emergency department visits have been shown to increase greatly with age among the young-old (65-74 years), middle-old (75-84 years), and old-old (85+ years) age groups [3]. The highest rate of hospitalizations in this study occurred in the old-old age group at $66.5 \%$; the overall hospitalization rate for elderly patients was $57.1 \%$. Incidence of infection increases as patients age, with the oldold age group having $>30$ cases $/ 100,000$ persons [4].

In a study by Solis-Hernandez et al., which compared hospital-acquired infections in the elderly $(65+$ years $)$ versus younger patients, the elderly population demonstrated a statistically significant increase in acquisition of ventilator-associated pneumonia, secondary bacteremia, and catheter- 
associated urinary tract infections (UTIs) [5]. The elderly patients in this study also experienced similar rates of hospitalacquired pneumonia, primary bacteremia, catheter-related bloodstream infections, UTIs, surgical site infections, and skin and soft tissue infections (SSTIs). Methicillin-resistant Staphylococcus aureus (MRSA) is often suspected in these hospital-acquired infections. The drug of choice to provide empiric antibiotic coverage of MRSA in these infections is frequently vancomycin.

Vancomycin is a glycopeptide antibiotic first discovered in the 1950s and approved by the FDA in 1958 [6]. It has slowly bactericidal activity against Gram-positive organisms such as Staphylococcus aureus and pneumococci, and bacteriostatic activity against enterococci [7, 8]. Vancomycin also has activity against Clostridium difficile [7]. Early on, toxicity fears limited the uptake of vancomycin in favor of emerging semi-synthetic penicillins and cephalosporins [6]. Toxicities associated with vancomycin include infusion-related reactions (including red man syndrome), nephrotoxicity, and ototoxicity. Purification of vancomycin over the years has resulted in fewer toxicities. Use of vancomycin greatly increased in the 1980s and 1990s, with the emergence of MRSA. Currently, vancomycin is one of the primary antibiotics used to treat complicated infections suspected to be caused by MRSA, including nosocomial infections [8]. According to the guidelines set forth by the Infectious Diseases Society of America (IDSA), in patients with normal renal function, a vancomycin dose of 15-20 mg/ $\mathrm{kg}$ of actual body weight every $8-12 \mathrm{~h}$ is recommended, not to exceed $2 \mathrm{~g}$ per dose [9]. In critically ill patients, a loading dose of $25-30 \mathrm{mg} / \mathrm{kg}$ may be considered. In patients with compromised renal function, dosing intervals should be extended to every $24 \mathrm{~h}$ or greater. Serum trough concentrations should be monitored prior to the fourth dose (approximate steady-state concentration). Goal trough values depend on the source of infection; for serious infections, troughs of $15-20 \mu \mathrm{g} / \mathrm{mL}$ should be targeted [8]. IDSA guidelines recommend vancomycin as first-line therapy for complicated SSTIs, bacteremia, infective endocarditis, pneumonia, osteomyelitis, septic arthritis, meningitis, and abscesses [8]. While the guidelines provide dosing recommendations specific for the pediatric population, there are no recommendations specific for the geriatric population, other than dosing based an individual's creatinine clearance.

\section{Pharmacokinetic and Pharmacodynamic Considerations in the Elderly}

With creatinine clearance representing the only guidance for vancomycin dosing in the elderly population, it is important to consider the pharmacokinetic and pharmacodynamic factors that may be altered in these patients.

\subsection{Pharmacokinetics}

\subsubsection{Distribution}

There are multiple physiological aspects that can affect the distribution of drugs, particularly in the elderly, including the extent of protein binding and total body weight. Albumin, the most abundant plasma protein that drugs bind to, can be utilized as a marker for malnutrition. Vancomycin displays approximately $50 \%$ protein binding, primarily to albumin, in normal, healthy adults [10]. While age may not be directly correlated to hypoalbuminemia, the elderly patient population has alterations in taste and smell which have been associated with geriatric anorexia, ultimately leading to hypoalbuminemia secondary to malnutrition [11-14]. In patients 60 years or older, a reduction in albumin of up to $25 \%$ was observed compared with patients $<40$ years of age [15]. Furthermore, in the community, approximately one in ten elderly adults suffers from malnutrition [16]. Conversely, malnutrition rates are high, up to $61 \%$, in elderly hospitalized patients compared with the general medicine hospitalized population with rates of $11-44 \%$ [17]. An analysis of risk factors for long-term ( $\geq 14$ days) anti-MRSA therapy identified albumin $\leq 2.5 \mathrm{~g} /$ $\mathrm{dL}$ as an independent risk factor in a cohort of 71 patients [18]. A study evaluating the impact of albumin on vancomycin in patients 75 years or older with MRSA pneumonia demonstrated worse clinical outcomes for patients with severe $(<2.5 \mathrm{~g} / \mathrm{dL})$ versus non-severe hypoalbuminemia [19]. Severe hypoalbuminemia was associated with increased 28-day mortality, and nephrotoxicity was associated with an increase in vancomycin half-life. However, it is important to note that these patients had higher severity of infection at baseline.

Total body weight also affects the volume of distribution. Irrespective of age, obesity $(\mathrm{BMI} \geq 30)$ rates continue to rise over time in the United States [20]. In the elderly specifically, rates of obesity differ based upon age classification. In the young-old (65-74 years of age) population, obesity rates are highest at $40.8 \%$, affecting over 8 million US adults [20]. Fortunately, this number decreases dramatically to $27.8 \%$ in the middle- and old-old populations. Patient weight is an important characteristic when considering vancomycin dosing because total body weight is directly correlated to volume of distribution of this agent $[21,22]$. Pending weight status, over- or under-dosing of vancomycin could easily occur.

\subsubsection{Metabolism}

Vancomycin does not undergo apparent metabolism; therefore, age would not likely affect this pharmacokinetic parameter. 


\subsubsection{Excretion/Elimination}

With renal function being the primary route of elimination for intravenous vancomycin, changes related to aging are an important consideration. Although the half-life $\left(t_{1 / 2}\right)$ of vancomycin is $4-6 \mathrm{~h}$ in healthy subjects with normal renal function [10], decreased renal function has repeatedly demonstrated an inverse correlation to elimination half-life [23-28]. In an evaluation of 288 hospitalized patients (148 elderly, 140 young) receiving vancomycin therapy, the mean $t_{1 / 2}$ observed was 17.8 versus $7.5 \mathrm{~h}$, respectively [29]. Without dosage adjustments, there is an increased risk of vancomycin-induced nephrotoxicity in the elderly population due to prolonged $t_{1 / 2}$, likely attributable to decreased renal function.

\subsection{Pharmacodynamics}

Comparable with the non-elderly population, the pharmacodynamic parameter that best correlates with efficacy for vancomycin is the ratio of the 24-h area under the concentration-time curve and minimum inhibitory concentration $\left(\mathrm{AUC}_{0-24} / \mathrm{MIC}\right)[9,30]$. While an AUC/MIC $>400$ has been established as the number needed for treatment efficacy [31], emerging data suggests that an AUC/MIC of $\geq 521$ for dose 1 and $\geq 650$ for dose 2 are more accurate at maintaining treatment efficacy [32]. Despite the AUC/MIC more closely associating with clinical success than serum trough concentrations, it is rarely calculated due to perceived difficulty for bedside clinicians $[31,33]$. Therefore, vancomycin serum trough concentrations of $15-20 \mu \mathrm{g} / \mathrm{mL}$ are utilized as a surrogate marker for clinical success $[34,35]$. Unfortunately, clinical failure, including mortality, is more likely when the MRSA MIC values to vancomycin are $>1 \mu \mathrm{g} / \mathrm{mL}$ due to the inability to achieve the appropriate AUC/MIC target [36-38]. Despite numerous reports demonstrating this finding, one meta-analysis assessing the association between vancomycin MIC and mortality in patients with $S$. aureus bacteremia did not find a mortality association [39]. The authors of this study concluded that clinicians should ensure that patients are evaluated for proper source control and treated for an appropriate duration regardless of vancomycin MIC values. Conversely, other studies have demonstrated that treatment failure is more likely for organisms with vancomycin MICs $>1 \mu \mathrm{g} / \mathrm{mL}$, even when agents other than vancomycin are utilized, suggesting that these strains may have increased virulence when compared with strains with lower MICs [40-42]. Therefore, in instances in which there is an isolate with an elevated vancomycin MIC $(>1 \mu \mathrm{g} / \mathrm{mL})$, vancomycin should be avoided.

\section{Key Clinical Issues}

\subsection{Identifying Infections}

Elderly adults continue to be at high risk for infections, leading to greater complications and subsequent mortality, than younger counterparts [43-45]. Some factors that contribute to this increased risk include weakened immune systems, increased prevalence of underlying comorbidities, increased amount of diagnostic and invasive procedures, and decreased physiologic reserves to fight infection [43, 44]. The most common infections in elderly adults tend to be respiratory tract infections, UTIs, abdominal infections, and SSTIs [44]. Respiratory tract infections, specifically pneumonia, are the eighth leading cause of death in the US [46], with close to $90 \%$ of these occurring in adults 65 years of age and older [43]. Especially concerning is the lack of classic clinical manifestations in elderly adults. These patients may present with weakness, confusion, or urinary incontinence [44], instead of traditional signs such as fever and leukocytosis. Additionally, elderly patients, especially adults in institutional settings, are at an increased risk of colonization and subsequent infection with MRSA [43, 45, 47] at a rate that is five times higher than in younger individuals [48]. It is important to recognize these non-traditional signs of infection to be able to adequately care for this population.

\subsection{Estimating Renal Function and Dosing Vancomycin}

Renal function declines with age due to functional and structural changes [49], and dosage adjustments for renal function are a necessity. Elderly patients represent a heterogeneous population [50], thus making it difficult to accurately estimate their renal function. Studies have examined several equations in an attempt to elucidate an accurate estimate of renal function: the Cockcroft-Gault (CG) method, the Modification of Diet in Renal Disease (MDRD) method, and the Chronic Kidney Disease Epidemiology Collaboration (CKD-EPI) method. Each of these methods is derived from different patient characteristics, but all only provide estimates of the creatinine clearance. Elderly patients have characteristics that can influence interpretation and use of these equations, such as increased comorbidities, decreased nutritional status, and lower body fat to muscle mass composition [51]. Additionally, many elderly adults may have decreased muscle mass, which also leads to lower serum creatinine [49, 52]. However, they also can have serum creatinine values within the normal limits but still have reduced renal function [53]. If a lower serum creatinine value is utilized 
to estimate renal function, this can result in overestimation of doses required for renally eliminated drugs, thus contributing to drug overdose and toxicity [54]. This has led many clinicians to compensate for a low serum creatinine by rounding up to $1 \mathrm{mg} / \mathrm{dL}$; however, this practice has not been validated and is not encouraged [52, 55]. A study by Roberts et al. examined the differences between the MDRD equation, the traditional CG equation, and an optimized CG equation in determining which provided a more accurate assessment of renal function in elderly patients to adjust drug doses [54]. The optimized CG equation differs from the traditional CG equation by utilizing the lesser of actual body weight or ideal body weight and capping the serum creatinine at a minimum of $0.68 \mathrm{mg} / \mathrm{dL}$, instead of $1 \mathrm{mg} /$ dL. Additionally, the authors measured creatinine values using assays that were aligned with the isotope dilutionmass spectrometry (IDMS) method to be able to standardize their results. The authors found that the traditional CG equation overestimated renal function, the optimized CG equation underestimated renal function by $10 \%$, and the MDRD equation overestimated renal function by $29 \%$. They concluded that the optimized CG equation was the most appropriate of the three equations examined to dose renally eliminated drugs. Conversely, a study by Tsuji et al. found that if the serum creatinine is measured using the Jaffe method, instead of the IDMS method, then a correction of serum creatinine to $0.68 \mathrm{mg} / \mathrm{dL}$ is unnecessary within the CG equation [56]. Additionally, a study by Glatard et al. found that the CG equation, MDRD equation, and CKD-EPI equations are not interchangeable when calculating vancomycin dosing and prediction of concentrations based on renal function [57]. The authors concluded that whichever equation clinicians utilize to calculate renal function should be the same equation used to dose renally eliminated drugs.

As vancomycin is approximately $90 \%$ renally eliminated unchanged in the urine [56,57], it is imperative to accurately estimate renal function when calculating dosing regimens to help optimize the pharmacokinetic and pharmacodynamic properties of the drug. Cutler et al. compared vancomycin kinetics in younger patients (mean age: 23 years) versus older patients (mean age: 68 years) [58]. They concluded that there was a significant difference in vancomycin distribution between younger patients $(0.64 \mathrm{~L} /$ $\mathrm{kg})$ and older patients $(0.93 \mathrm{~L} / \mathrm{kg})$ that is not attributable to protein binding, but rather to greater tissue binding. Furthermore, the authors found that the vancomycin half-life was shorter in younger patients $(7.2 \mathrm{~h})$ than in elderly patients $(12.1 \mathrm{~h})$, which was attributable to the enlarged distribution (44\% higher than in younger patients) and reduced clearance (23\% lower than in younger patients). Another study by Sánchez et al. also found elderly patients to have an expanded volume of distribution and a reduced clearance than younger patients [59]. Hence, when determining dosing frequency, it is vital to account for the volume of distribution, target trough, elimination rate, and duration of therapy. The target trough range for vancomycin is anywhere between 10 and $20 \mu \mathrm{g} / \mathrm{mL}$, depending on the site of infection. In elderly adults, it is recommended to utilize lower empiric maintenance doses of vancomycin to compensate for the reduced clearance secondary to enhanced tissue binding and reduced overall systemic and renal clearance $[44,58]$. Increased duration of vancomycin therapy has been linked to the development of nephrotoxicity; therefore, close monitoring of vancomycin concentrations and renal function is highly recommended in elderly patients [60].

Elderly patients who are critically ill represent a unique challenge when dosing vancomycin, not only due to altered pharmacodynamics, but also because of a paucity of data available. A recent study by Shahrami et al. examined the impact of early individualized dosing of vancomycin in critically ill patients (mean age: 48 years) compared with standard monitoring [61]. They concluded that by monitoring serum concentrations of vancomycin obtained after the first dose, and again 4-6 h later, more patients were able to achieve goal peak and trough steady-state concentrations. While more data is needed to validate these findings, this study represents a valid option for pursuing optimization of vancomycin therapy in critically ill patients.

Another vancomycin optimization concept that has been introduced in recent years is continuous infusion. The intent of continuous infusion is to reduce toxicity, achieve target concentrations quicker, and avoid treatment failure [62]. Several studies have examined the impact of continuous infusion vancomycin in critically ill patients with mixed results. Saugel et al. conducted a prospective, observational study evaluating a dosing algorithm for continuous vancomycin administration in critically ill patients [63]. They found that continuous infusion of vancomycin allowed for rapid achievement of target serum concentrations but that these concentrations often became supratherapeutic. Blot et al. examined 42 patients in 26 different intensive care units (ICUs) from the Defining Antibiotic Levels in Intensive Care (DALI) study to determine if contemporary vancomycin dosing results in a favorable response [64]. They identified that while continuous infusion of vancomycin was able to attain clinically relevant trough concentrations and pharmacodynamic exposure, there was significant variation within the population, and the benefits of continuous infusion were not retained in multivariate analysis. When examining clinical outcomes of continuous infusion, Tafelski et al. found that while target serum vancomycin concentrations were achieved 1 day earlier in continuous infusion versus 
intermittent infusion, there were no significant differences in ICU mortality rate, duration of ICU stay, and duration of mechanical ventilation between groups [62].

While trying to achieve pharmacodynamic targets for vancomycin administration, clinicians also need to balance the incidence of nephrotoxicity. A study by Cianferoni et al. examined the incidence of nephrotoxicity in 207 critically ill patients receiving continuous-infusion vancomycin [65]. The authors concluded that $25 \%$ of the included patients developed acute kidney injury, which was predicted by the magnitude of vancomycin serum concentrations and duration of vancomycin therapy. Another study by Hanrahan et al. examined the incidence of nephrotoxicity and death based on continuous versus intermittent vancomycin infusion strategies [66]. The authors also found that the incidence of nephrotoxicity was related to the magnitude of vancomycin serum concentrations and duration of vancomycin therapy. While there were numerically more patients in the continuous infusion group that developed nephrotoxicity than in the intermittent infusion group (54 vs $26 \%$ ), a multivariate regression model indicated that intermittent infusion, among other factors, was an independent predictor of the development of nephrotoxicity. The authors also concluded that despite having a higher incidence of death within $72 \mathrm{~h}$ of the last vancomycin dose in the continuous infusion group compared with the intermittent infusion group (20 vs $9 \%$ ), there was no statistically significant difference in mortality. Overall, controversy still remains as to the clinical efficacy and utility of employing continuous infusion vancomycin in critically ill patients. Additionally, the studies mentioned above did not examine the impact of continuous infusion vancomycin in elderly critically ill patients, making the generalization to this population difficult.

\subsection{Vancomycin Monitoring and Infection Resolution}

When utilizing vancomycin, it is important to ensure safe and effective concentrations of the drug. Vancomycin has been shown to have a prolonged half-life in elderly patients compared with younger patients, as well as a larger volume of distribution and decreased clearance, which are all factors that can contribute to vancomycin accumulation [56-58]. With accumulation of vancomycin comes the risk for its most concerning side effect, which is the incidence of nephrotoxicity. Current estimates of vancomycin-induced nephrotoxicity range anywhere from 5 to $35 \%$ [60], with some sources citing a specific incidence of $31-34 \%$ in patients 65 years of age and older [67]. As previously discussed, the compromised renal function found in elderly patients often puts them at increased risk of developing nephrotoxicity due to vancomycin [60]; however, there are also data that suggest that increased age alone (in the absence of other complications) is not a risk factor for vancomycin-induced nephrotoxicity [67].

Once adequate therapy has been prescribed, it is important to monitor for signs of resolving infection. Elderly patients tend to have greater complications from infections, leading to increased morbidity and mortality [44]. Additionally, the goal may not always be to return elderly patients to a normal functioning state, but rather, to return them to their baseline functional status. Thus, it is extremely important to identify what the end goal of therapy needs to be for these patients.

\section{Practical Applications}

While dosing of vancomycin should be individualized for each patient, there is a general thought process for healthcare providers when dosing vancomycin in elderly patients (Fig. 1).

\subsection{Dosing}

When dosing vancomycin in elderly patients, one should consider the entire patient. What is the body mass composition? Is the patient frail or healthy? Patients who are healthy, especially in the young-old and sometimes in the middle-old subsets, often can be initiated on treatment dosages the same as in a younger adult $(15-20 \mathrm{mg} / \mathrm{kg}$ with frequency dependent on renal function) [9]. As patients become frail, lose muscle mass, or approach the old-old subset, alterations in the dosage or dosing frequency may be necessary regardless of age or laboratory values.

For elderly patients who are not as healthy as younger adults, consideration should be given to a reduction in maintenance dosing. Vancomycin is highly lipophilic and distributes well into tissue, including fat [10]. Accordingly, patients with less body fat may concentrate vancomycin more rapidly in the blood stream, potentially leading to a higher risk of toxicity secondary to accumulation. In patients who are frail or thin, loading doses should still be initiated at $25-30 \mathrm{mg} / \mathrm{kg}$ based on actual body weight, especially if the actual body weight is less than the ideal body weight, in patients with severe infections from suspected MRSA [9]. If practicing at an institution that rounds vancomycin doses for ease of preparation, consideration should be given to rounding down to the nearest increment instead of rounding up, depending on severity of illness. Maintenance doses may be empirically reduced (one study suggested $10 \mathrm{mg} / \mathrm{kg}$ ) and frequency adjusted for renal function in these patients [68].

Similarly, patients in the middle-old or old-old subset who are obese may also need altered dosing regimens 
Vancomycin Ordered

\begin{tabular}{|c|c|c|}
\hline & & Vancomycin Ordered \\
\hline Step 1: & $\begin{array}{l}\text { Check the following information: } \\
\text { - Indication for therapy } \\
\text { - } \quad \text { Patient characteristics } \\
\text { - } \quad \text { Culture results } \\
\quad \text { Severity of illness }\end{array}$ & $\begin{array}{l}\text { (pneumonia, endocarditis, skin and skin structure infection, etc) } \\
\text { (age, height, weight, serum creatinine) } \\
\text { (pending, confirmed MRSA, suspected MRSA) } \\
\text { (intensive care unit, general medicine floor, use of vasopressors or mechanical ventilation) }\end{array}$ \\
\hline Step 2: & $\begin{array}{l}\text { Assess the following information: } \\
\text { - Target trough or target AUC:MIC rat } \\
\text { - Renal dose adjustments } \\
\text { - Drug-drug interactions (concomitan } \\
\text { - } \text { Allergies or intolerances (Red Man }\end{array}$ & $\begin{array}{l}\text { io } \\
\text { t nephrotoxins) } \\
\text { yndrome) }\end{array}$ \\
\hline Step 3: & $\begin{array}{l}\text { Calculate the following information: } \\
\text { - } \quad \text { Loading dose (if needed) } \\
\quad \text { Empiric maintenance dose using }\end{array}$ & opulation-based pharmacokinetic equations \\
\hline Step 4: & $\begin{array}{l}\text { Recognize the following monitoring the } \\
\text { - Vancomycin concentrations (peak, } t \\
\text { - Renal function (creatinine clearance }\end{array}$ & $\begin{array}{l}\text { t needs to be performed: } \\
\text { rough, and/or AUC:MIC) }\end{array}$ \\
\hline Step 5: & 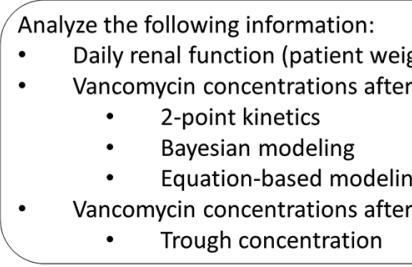 & $\begin{array}{l}\text { int, serum creatinine, urine output) } \\
\text { first dose } \\
\text { steady state }\end{array}$ \\
\hline Step 6: & $\begin{array}{l}\text { Adjust the following information: } \\
\text { - Maintenance dose, as needed, to ac }\end{array}$ & hieve target trough concentrations or target AUC:MIC ratio \\
\hline Step 7: & $\begin{array}{l}\text { Monitor the following information: } \\
\text { - Signs and symptoms of clinical impr } \\
\text { - Signs and symptoms of vancomycin }\end{array}$ & $\begin{array}{l}\text { ovement } \\
\text { toxicity }\end{array}$ \\
\hline Step 8: & $\begin{array}{l}\text { Determine the following information: } \\
\text { - } \quad \text { Appropriate duration of therapy }\end{array}$ & \\
\hline
\end{tabular}

Fig. 1 Steps to consider when dosing vancomycin in elderly patients. AUC/MIC the ratio of the 24-h area under the concentration-time curve and minimum inhibitory concentration, MRSA methicillin-resistant Staphylococcus aureus

$[69,70]$. In these patients, loading doses of $15-25 \mathrm{mg} / \mathrm{kg}$ should be based on actual body weight [71]. Because vancomycin accumulates in the tissues [58, 72], consideration should be given to empirically reducing the maintenance dose, particularly in patients who are critically ill $[9,29,68,70]$. Additionally, even though vancomycin is $50 \%$ bound to serum albumin [10], clinically dosing vancomycin according to serum albumin concentrations is not performed. Conversely, it is important to remember that elderly patients with low albumin are at risk for longer vancomycin therapy [18]. Furthermore, elderly patients with MRSA pneumonia also have the potential to have worse clinical outcomes despite adequate vancomycin therapy, in part due to low albumin [19]. Therefore, if practicing at an institution that utilizes loading doses, consider a lower empiric maintenance dose to account for the serum accumulation over time, while still adjusting further maintenance dosing by utilizing serum concentrations or calculated AUC/MIC ratio of vancomycin $[29,68,73]$.
Regardless of weight, clinicians should carefully consider age-related changes in kidney function, including loss of $20-25 \%$ of renal mass and $20-30 \%$ of glomeruli compared with younger patients [74]. In addition to renal changes, muscle mass often decreases with increasing age [75]. This can impact laboratory values such as serum creatinine, ultimately impacting common calculations used to estimate creatinine clearance (such as the CG equation) $[75,76]$. In patients with muscle tone similar to a younger adult, frequency of vancomycin should follow recommendations based on estimated glomerular filtration rate [10]. In frail patients or those with reduced muscle mass or suspected reductions in renal function, clinicians may consider adjustments for very low serum creatinine values $(<0.5 \mathrm{mg} / \mathrm{dL})$, possibly including rounding to a higher value $(0.68 \mathrm{mg} / \mathrm{dL})$ in order to more accurately estimate creatinine clearance [54]. Because renal function is often decreased in elderly patients $[77,78]$, particularly the oldold subset, consideration should be given to empirically beginning these patients with daily administration instead 
of twice daily administration, regardless of estimated creatinine clearance.

\subsection{Determination of Response}

Irrespective of dosing strategy used, elderly patients should be monitored for evidence of response, including both efficacy and toxicity. In patients with stable renal function, vancomycin serum trough concentrations should be monitored just prior to the fourth dose for short courses, and adjustments made accordingly [9]. For longer courses, clinicians may choose to monitor routinely as deemed clinically appropriate [9]. Clinically, patients with stable renal function on prolonged courses are monitored approximately once per week. Goal trough concentrations for adults are generally between 15 and $20 \mu \mathrm{g} / \mathrm{mL}$, but may be higher or lower depending on infection, severity of illness, and clinical judgement [9]. In the elderly, new evidence suggests that troughs $>15 \mu \mathrm{g} / \mathrm{mL}$ are associated with increased rates of nephrotoxicity, and should not be exceeded [79]. Clinicians should monitor elderly patients carefully, possibly accepting a lower trough concentration if clinical improvement is seen.

In patients with fluctuating or rapidly changing renal function, clinicians should consider monitoring serum concentrations sooner than the fourth dose or more frequently [61]. If the trough concentration is drawn prior to the fourth dose, concentrations above the desired range likely signify potential for toxicity and may represent a need for a lower dose. Low concentrations will not be representative of the steady-state concentration and should be addressed using clinical judgement.

In recent literature, it has been suggested that the AUC/ MIC ratio may actually be a better predictor of the pharmacokinetics of vancomycin [80]. Although AUC/MIC ratios are not often used clinically because of the difficulty in calculation, trough concentrations are monitored as a surrogate for AUC/MIC ratio [9]. However, data by Pai et al. has demonstrated that single trough concentrations are not sufficient to aid clinicians in effectively dosing vancomycin [32]. The authors recommended two different approaches to monitoring vancomycin: Bayesian and equation-based. Bedside software programs are available for clinicians to be able to perform Bayesian modeling for patients on vancomycin, thus personalizing the patient's care. Alternatively, equation-based monitoring can be programmed into the electronic medical system to automatically calculate the patient's daily vancomycin AUC. Additionally, new evidence suggests that specific AUC/ MIC goals may be better clinical markers of therapeutic efficacy [73]. In the general adult population, AUC/MIC ratio of $>400 \mu \mathrm{gh} / \mathrm{mL}$ is targeted to provide effective therapy should the MRSA isolate have a vancomycin MIC $\leq 1$ [9]; alternatively, the new proposed AUC/MIC targets of $\geq 521$ on day 1 and $\geq 650$ on day 2 should be sufficient for a $80-85 \%$ clinical success rate [36]. In the elderly population, a targeted AUC/MIC of $250-450 \mu \mathrm{g} \mathrm{h} / \mathrm{mL}$ may provide improved efficacy while limiting toxicity [73]. Ultimately, the decision on how to monitor vancomycin and adjust doses lies with the clinician.

In addition to serum concentrations, clinicians should monitor for clinical resolution of infection or improvement in systemic symptoms (resolution of fever, normalization of white blood cell count, improvement in inflammatory biomarkers) in order to determine clinical response. If it is determined that the patient is not responding clinically after a sufficient trial of appropriately dosed vancomycin (appropriate trough concentrations for 5-7 days, depending on infection location and severity), consideration should be given to adjusting the dose or choosing an alternative treatment regimen.

\section{Conclusions}

Elderly patients have many physiologic changes that occur that put them at increased risk for infection, renally adjusted dosing miscalculations, and adverse drug reactions. It is important to remember that the volume of distribution increases, serum creatinine may decrease, increase, or remain constant, and creatinine clearance decreases in elderly patients. Loading doses remain consistent with recommendations for all patient ages, while lower maintenance doses and frequencies are recommended specifically in elderly patients. Monitoring of vancomycin concentrations earlier than steady state, as well as continued monitoring using either Bayesian forecasting or equation-based approaches should be considered for this patient population. Clinicians should understand the physiologic changes that occur, particularly when utilizing vancomycin to treat elderly patients with suspected or documented Gram-positive infections, in order to safely and effectively treat this patient population. Overall, elderly patients represent a heterogeneous group that should be treated individually and monitored closely.

\section{Compliance with Ethical Standards}

Statement of human rights For this type of review, formal consent is not required.

Funding This review was unfunded.

Conflict of interest KEB declares she has no conflict of interest. AMB declares she has no conflict of interest. KRS declares she has no conflict of interest. JLW declares she has no conflict of interest. 
Open Access This article is distributed under the terms of the Creative Commons Attribution-NonCommercial 4.0 International License (http://creativecommons.org/licenses/by-nc/4.0/), which permits any noncommercial use, distribution, and reproduction in any medium, provided you give appropriate credit to the original author(s) and the source, provide a link to the Creative Commons license, and indicate if changes were made.

\section{References}

1. Centers for Disease Control and Prevention. The state of aging and health in America 2013. Atlanta: Centers for Disease Control and Prevention, U.S. Department of Health and Human Services; 2013.

2. Videcnik Zorman J, Lusa L, Strle F, Maraspin V. Bacterial infection in elderly nursing home and community-based patients: a prospective cohort study. Infection. 2013;41:909-16.

3. Goto T, Yoshida K, Tsugawa Y, Camargo CA, Hasegawa K. Infectious disease-related emergency department visits of elderly adults in the United States, 2011-2012. J Am Geriatr Soc. 2016;64:31-6.

4. Edwards MS, Baker CJ. Group B streptococcal infections in elderly adults. Clin Infect Dis. 2005;41:839-47.

5. Solis-Hernandez PS, Vidales-Reyes M, Garza-Gonzalez E, Guajardo-Alvarez G, Chavez-Moreno S, Camacho-Ortiz A. Hospital-acquired infections in elderly versus younger patients in an acute care hospital. Int $\mathbf{J}$ Infect. 2016;3(1):e32620.

6. Levine DP. Vancomycin: a history. Clin Infect Dis. 2006;42(Suppl 1):S5-12.

7. French GL. Bactericidal agents in the treatment of MRSA infections - the potential role of daptomycin. J Antimicrob Chemother. 2006;58:1107-17.

8. Liu C, Bayer A, Cosgrove SE, et al. Clinical practice guidelines by the Infectious Diseases Society of America for the treatment of methicillin-resistant Staphylococcus aureus infections in adults and children. Clin Infect Dis. 2011;52:1-38.

9. Rybak M, Lomaestro B, Rotschafer JC, et al. Therapeutic monitoring of vancomycin in adult patients: a consensus review of the American Society of Health-System Pharmacists, the Infectious Diseases Society of America, and the Society of Infectious Diseases Pharmacists. Am J Health System Pharm. 2009;66(1):82-98.

10. Product Information: vancomycin $\mathrm{HCl}$ IV injection, vancomycin HCl IV injection. Lake Forest: Hospira, Inc. (per manufacturer); 2015.

11. Cabrerizo S, Cuadras D, Gomez-Busto F, Artaza-Artabe I, MarínCiancas F, Malafarina V. Serum albumin and health in older people: review and meta-analysis. Maturitas. 2015;81(1):17-27.

12. Seiler WO. Clinical pictures of malnutrition in ill elderly subjects. Nutrition. 2001;17(6):496-8.

13. Volkert D, Berner YN, Berry E, et al. ESPEN guidelines on enteral nutrition: geriatrics. Clin Nutr. 2006;25(2):330-60.

14. Malafarina V, Uriz-Otano F, Gil-Guerrero L, Iniesta R. The anorexia of ageing: physiopathology, prevalence, associated comorbidity and mortality. A systematic review. Maturitas. 2013;74(4):293-302.

15. Hayes MJ, Langman MJ, Short AH. Changes in drug metabolism with increasing age: 2 . phenytoin clearance and protein binding. Br J Clin Pharmacol. 1975;2(1):73-9.

16. Wilson L. A review and summary of the impact of malnutrition in older people and the report costs and benefits of interventions. Malnutrition Task Force. 2013. Available at http://www. malnutritiontaskforce.org.uk. Accessed 5 June 2016.

17. Corish CA, Kennedy NP. Protein-energy undernutrition in hospital in-patients. Br J Nutr. 2000;83(6):575-91.
18. Hayashi H, Matsuzaki T, Mizuno Y, et al. Analysis of factors affecting long-term administration of anti-methicillin resistant Staphylococcus aureus (MRSA) drugs. Yakugaku Zasshi. 2009;129(3):347-52.

19. Mizuno T, Mizokami F, Fukami K, et al. The influence of severe hypoalbuminemia on the half-life of vancomycin in elderly patients with methicillin-resistant Staphylococcus aureus hospital-acquired pneumonia. Clin Interv Aging. 2013;8:1323-8.

20. Fakhouri T, Ogden CL, Carroll MD, Kit BK, Flegal KM. Prevalence of obesity among older adults in the United States, 2007-2010. Natl Center Health Stat Data Brief. 2012;106:1-8.

21. Grace E. Altered vancomycin pharmacokinetics in obese and morbidly obese patients: what we have learned over the past 30 years. J Antimicrob Chemother. 2012;67(6):1305-10.

22. Blouin RA, Bauer LA, Miller DD, Record KE, Griffen WO Jr. Vancomycin pharmacokinetics in normal and morbidly obese subjects. Antimicrob Agents Chemother. 1982;21(4): 575-80.

23. Matzke GR, McGory RW, Halstenson CE, Keane WF. Pharmacokinetics of vancomycin in patients with various degrees of renal function. Antimicrob Agents Chemother. 1984;25:433-7.

24. Moellering RC Jr, Krogstad DJ, Greenblatt DJ. Vancomycin therapy in patients with impaired renal function: a nomogram for dosage. Ann Intern Med. 1981;94:343-6.

25. Rodvold KA, Blum RA, Fischer JH, et al. Vancomycin pharmacokinetics in patients with various degrees of renal function. Antimicrob Agents Chemother. 1988;32:848-52.

26. Ducharme MP, Slaughter RL, Edwards DJ. Vancomycin pharmacokinetics in a patient population: effect of age, gender, and body weight. Ther Drug Monit. 1994;16:513-8.

27. Birt JK, Chandler MH. Using clinical data to determine vancomycin dosing parameters. Ther Drug Monit. 1990;1990(12) :206-9.

28. Murphy JE, Gillespie DE, Bateman CV. Predictability of vancomycin trough concentrations using seven approaches for estimating pharmacokinetic parameters. Am J Health Syst Pharm. 2006;63:2365-70.

29. Guay DR, Vance-Bryan K, Gilliland S, Rodvold K, Rotschafer J. Comparison of vancomycin pharmacokinetics in hospitalized elderly and young patients using a Bayesian forecaster. J Clin Pharmacol. 1993;33(10):918-22.

30. Moise-Broder PA, Forrest A, Birmingham MC, Schentag JJ. Pharmacodynamics of vancomycin and other antimicrobials in patients with Staphylococcus aureus lower respiratory tract infections. Clin Pharmacokinet. 2004;43(13):925-42.

31. Lodise TP, Drusano GL, Zasowski E, et al. Vancomycin exposure in patients with methicillin-resistant Staphylococcus aureus bloodstream infections: how much is enough? Clin Infect Dis. 2014;59:666-75.

32. Pai MP, Neely M, Rodvold KA, Lodise TP. Innovative approaches to optimizing the delivery of vancomycin in individual patients. Adv Drug Deliv Rev. 2014;77:50-7.

33. Homes NE, Turnidge JD, Munckhof WJ, et al. Vancomycin AUC/MIC ratio and 30-day mortality in patients with Staphylococcus aureus bacteremia. Antimicrob Agents Chemother. 2013; 57(4):1654-63.

34. Song KH, Kim HB, Kim HS, et al. Impact of area under the concentration-time curve to minimum inhibitory concentration ratio on vancomycin treatment outcomes in methicillin-resistant Staphylococcus aureus bacteraemia. Int J Antimicrob Agents. 2015;46(6):689-95.

35. Men $\mathrm{P}$, Li HB, Zhai SD, Zhao RS. Association between the $\mathrm{AUC}_{0-24} / \mathrm{MIC}$ ratio of vancomycin and its clinical effectiveness: a systematic review and meta-analysis. PLoS One. 2016; 11(1):e0146224. 
36. van Hal SJ, Lodise TP, Paterson DL. The clinical significance of vancomycin minimum inhibitory concentration in Staphylococcus aureus infections: a systematic review and meta-analysis. Clin Infect Dis. 2012;54(6):755-71.

37. Mavros MN, Tansarli GS, Vardakas KZ, et al. Impact of vancomycin minimum inhibitory concentration on clinical outcomes of patients with vancomycin-susceptible Staphylococcus aureus infections. Int J Antimicrob Agents. 2012;40(6):496-509.

38. Jacob JT, Diaz Granados CA. High vancomycin minimum inhibitory concentration and clinical outcomes in adults with methicillin-resistant Staphylococcus aureus infections. Int J Infect Dis. 2013;17(2):e93-100.

39. Kalil AC, Van Schooneveld TC, Fey PD, Rupp ME. Association between vancomycin minimum inhibitory concentration and mortality among patients with Staphylococcus aureus bloodstream infections: a systematic review and meta-analysis. JAMA. 2014;312(15):1552-64.

40. Holmes NE, Turnidge JD, Munckhof WJ, et al. Antibiotic choice may not explain poorer outcomes in patients with Staphylococcus aureus bacteremia and high vancomycin minimum inhibitory concentrations. J Infect Dis. 2011;204(3):340-7.

41. Cervera C, Castaneda X, de la Maria CG, et al. Effect of vancomycin minimum inhibitory concentration on the outcome of methicillin-susceptible Staphylococcus aureus endocarditis. Clin Infect Dis. 2014;58(12):1668-75.

42. San-Juan R, Viedma E, Chaves F, et al. High MICs for vancomycin and daptomycin and complicated catheter-related bloodstream infections with methicillin-sensitive Staphylococcus aureus. Emerg Infect Dis. 2016;22(6):1057-66.

43. Mouton CP, Bazaldua OV, Pierce B, Espino DV. Common infections in older adults. Am Fam Physician. 2001;63:257-68.

44. Rajagopalan S, Yoshikawa TT. Antimicrobial therapy in the elderly. Med Clin N Am. 2001;85(1):133-47.

45. McCollum M, Sorensen SV, Liu LZ. A comparison of costs and hospital length of stay associated with intravenous/oral linezolid or intravenous vancomycin treatment of complicated skin and soft-tissue infections caused by suspected or confirmed methicillin-resistant Staphylococcus aureus in elderly US patients. Clin Ther. 2007;29(3):469-77.

46. Xu J, Murphy SL, Kochanek KD, Bastian BA. Deaths: final data for 2013. National Vital Statistics Report, vol 64, no 2. Hyattsville: National Center for Health Statistics; 2016.

47. McClelland RS, Fowler VG, Sanders LL, et al. Staphylococcus aureus bacteremia among elderly vs younger adult patients. Arch Intern Med. 1999;159:1244-7.

48. Tacconelli E, Pop-Vicas AE, D'Agata EMC. Increased mortality among elderly patients with meticillin-resistant Staphylococcus aureus bacteraemia. J Hosp Infect. 2006;64:251-6.

49. Mitchell SCM, Sheldon TA, Shaw AB. Quantification of proteinuria: a re-evaluation of the protein/creatinine ratio for elderly subjects. Age Ageing. 1993;22(6):443-9.

50. Bourguignon L, Cazaubon Y, Debeurme G, Loue C, Ducher M, Goutelle $S$. Pharmacokinetics of vancomycin in elderly patients aged over 80 years. Antimicrob Agents Chemother. 2016;80(8):4563-7.

51. Spruill WJ, Wade WE, Cobb HH III. Comparison of estimated glomerular filtration rate with estimated creatinine clearance in the dosing of drugs requiring adjustments in elderly patients with declining renal function. Am $\mathrm{J}$ Geriatr Pharmacother. 2008;6:153-60.

52. Wilhelm SM, Kale-Pradhan PB. Estimating creatinine clearance: a meta-analysis. Pharmacotherapy. 2011;31(7):658-64.

53. Wasén E, Isoaho R, Mattila K, Vahlberg T, Kivelä SL, Irjala K. Estimation of glomerular filtration rate in the elderly: a comparison of creatinine-based formulae with serum cystatin C. J Intern Med. 2004;256:70-8.
54. Roberts GW, Ibsen PM, Schiøler CT. Modified diet in renal disease method overestimates renal function in selected elderly patients. Age Ageing. 2009;38:698-703.

55. Bertino JS Jr. Measured versus estimated creatinine clearance in patients with low serum creatinine values. Ann Pharmacother. 1993;27:1439-42.

56. Tsuji Y, Hiraki Y, Mizoguchi A, et al. Effect of various estimates of renal function on prediction of vancomycin concentration by the population mean and Bayesian methods. J Clin Pharm Ther. 2009;34:465-72.

57. Glatard A, Bourguignon L, Jelliffe RW, Maire P, Neely MN, Goutelle S. Influence of renal function estimation on pharmacokinetic modeling of vancomycin in elderly patients. Antimicrob Agents Chemother. 2015;59(6):2986-94.

58. Cutler NR, Narang PK, Lesko LJ, Ninos M, Power M. Vancomycin disposition: the importance of age. Clin Pharmacol Ther. 1984;36(6):803-10.

59. Sánchez JL, Dominguez AR, Lane JR, Anderson PO, Capparelli EV, Cornejo-Bravo JM. Population pharmacokinetics of vancomycin in adult and geriatric patients: comparison of eleven approaches. Int J Clin Pharmacol Ther. 2010;48(8):525-33.

60. Elyasi S, Khalili H, Dashti-Khavidaki S, Mohammadpour A. Vancomycin-induced nephrotoxicity: mechanism, incidence, risk factors and special populations: a literature review. Eur J Clin Pharmacol. 2012;68:1243-55.

61. Shahrami B, Najmeddin F, Mousavi S, et al. Achievement of vancomycin therapeutic goals in critically ill patients: early individualization may be beneficial. Crit Care Res Prac. 2016;2016(2016):Article ID 1245815.

62. Tafelski S, Nachtigall I, Troeger U, et al. Observational clinical study on the effects of different dosing regimens on vancomycin target levels in critically ill patients: continuous versus intermittent application. J Infect Public Health. 2015;8:355-63.

63. Saugel B, Gramm C, Wagner JY, et al. Evaluation of a dosing regimen for continuous vancomycin infusion in critically ill patients: an observational study in intensive care unit patients. J Crit Care. 2014;29:351-5.

64. Blot S, Koulenti D, Akova M, et al. Does contemporary vancomycin dosing achieve therapeutic targets in a heterogenous clinical cohort of critically ill patients?: data from the multinational DALI study. Crit Care. 2014;18:R99.

65. Cianferoni S, Devigili A, Ocampos-Martinez E, et al. Development of acute kidney injury during continuous infusion of vancomycin in septic patients. Infection. 2013;41:811-20.

66. Hanrahan TP, Harlow G, Hutchinson J, et al. Vancomycin-associated nephrotoxicity in the critically ill: a retrospective multivariate regression analysis. Crit Care Med. 2014;42(12):2527-36.

67. Carreño JJ, Jaworski A, Kenney RM, Davis SL. Comparative incidence of nephrotoxicity by age group among adult patients receiving vancomycin. Infect Dis Ther. 2013;2:201-8.

68. Kosmisky DE, Griffiths CL, Templin MA, Norton J, Martin KE. Evaluation of a new vancomycin dosing protocol in morbidly obese patients. Hosp Pharm. 2015;50(9):789-97.

69. Kubiak DW, Alquwaizani M, Sansonetti D, Barra ME, Calderwood MS. An evaluation of systemic vancomycin dosing in obese patients. Open Forum Infect Dis. 2015;2(4):ofv176.

70. Lin H, Yeh DD, Levine AR. Daily vancomycin dose requirements as a continuous infusion in obese versus non-obese SICU patients. Crit Care. 2016;20(1):205.

71. Vance-Bryan K, Guay DR, Gilliland SS, Rodvold KA, Rotschafer JC. Effect of obesity on vancomycin pharmacokinetic parameters as determined by using a Bayesian forecasting technique. Antimicrob Agents Chemther. 1993;37(3):436-40.

72. Fujiwara K, Yoshizaki Y, Shin M, Miyazaki T, Saita T, Nagata S. Immunocytochemistry for vancomycin using a monoclonal 
antibody that reveals accumulation of the drug in rat kidney and liver. Antimicrob Agents Chemother. 2012;56(11):5883-91.

73. Mizokami F, Shibasaki M, Yoshizue Y, Noro T, Mizuno T, Furuta K. Pharmacodynamics of vancomycin in elderly patients aged 75 years or older with methicillin-resistant Staphylococcus aureus hospital-acquired pneumonia. Clin Interv Aging. 2013;8:1015-21.

74. Garasto S, Fusco S, Corica F, et al. Estimating glomerular filtration rate in older people. BioMed Res Int. 2014;Article ID 916542:12.

75. Odden MC, Shlipak MG, Tager IB. Serum creatinine and functional limitation in elderly persons. J Cerontol A Biol Sci Med Sci. 2009;64A(3):370-6.

76. Global $\mathrm{RPh}$. Creatinine clearance methods. Global $\mathrm{RPh}$, Inc. Available at http://www.globalrph.com. Accessed 15 July 2016.
77. Gekle M. Kidney and aging- a narrative review. Exp Gerontol. 2016. doi:10.1016/j.exger.2016.03.013 (Epub ahead of print).

78. Bolignano D, Mattace-Raso F, Sijbrands EJ, Zoccali C. The aging kidney revisited: a systematic review. Ageing Res Rev. 2014;14:65-80.

79. Fukumori S, Tsuji Y, Mizoquchi A, Kasai H, Ishibashi T, Iwamura N, To H. Association of the clinical efficacy of vancomycin with the novel pharmacokinetic parameter area under the trough level (AUTL) in elderly patients with hospital-acquired pneumonia. J Clin Pharm Ther. 2016;41(4):399-402.

80. Prybylski JP. Vancomycin trough concentration as a predictor of clinical outcomes in patients with Staphylococcus aureus bacteremia: a meta-analysis of observational studies. Pharmacotherapy. 2015;35(10):889-98. 